\title{
Conversational User Interfaces to support Astronauts in Extraterrestrial Habitats
}

\author{
Ana Rita Gonçalves Freitas \\ Alexander Schülke \\ Simon Glaser \\ Pitt Michelmann \\ Thanh Nguyen Chi \\ Lisa Schröder \\ Zahra Fadavi \\ Gaurav Talekar \\ Jette Ternieten \\ Akash Trivedi \\ Jana Wahls \\ Warda Masood \\ University of Bremen \\ Germany
}

\author{
Christiane Heinicke \\ Center of Applied Space Technology \\ and Microgravity (ZARM) \\ Germany
}

\author{
Johannes Schöning \\ University of St. Gallen \\ Switzerland
}

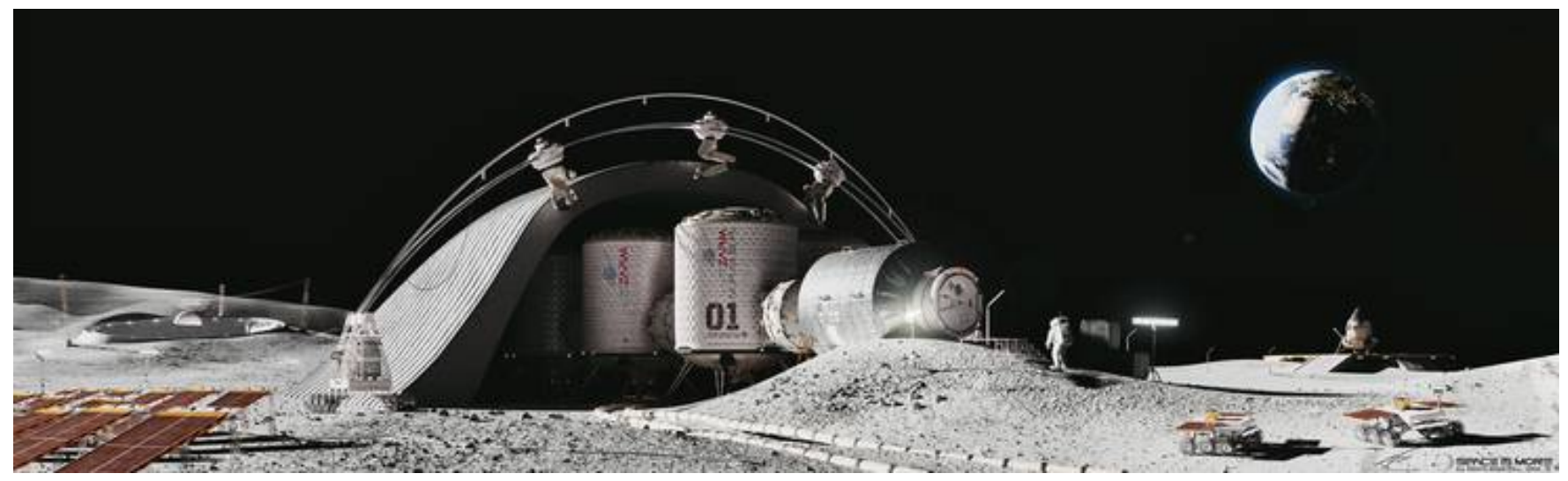

Figure 1: Artistic rendering of the MaMBA extraterrestrial habitat on the Moon.

\begin{abstract}
Long-term space missions are challenging and demanding for astronauts. Confined spaces and long-duration sensory deprivation may cause psychological problems for the astronauts. In this paper, we envision how extraterrestrial habitats (e.g., a habitat on the Moon or Mars) can maintain the well-being of the crews by augmenting the astronauts. In particular, we report on the design, implementation, and evaluation of conversational user interfaces
\end{abstract}

Permission to make digital or hard copies of all or part of this work for personal or classroom use is granted without fee provided that copies are not made or distributed for profit or commercial advantage and that copies bear this notice and the full citation on the first page. Copyrights for components of this work owned by others than the author(s) must be honored. Abstracting with credit is permitted. To copy otherwise, or republish, to post on servers or to redistribute to lists, requires prior specific permission and/or a fee. Request permissions from permissions@acm.org.

MUM 2021, December 5-8, 2021, Leuven, Belgium

(C) 2021 Copyright held by the owner/author(s). Publication rights licensed to ACM. ACM ISBN 978-1-4503-8643-2/21/05 . .\$15.00

https://doi.org/10.1145/3490632.3490673
(CUIs) for extraterrestrial habitats. The goal of such CUIs is to support scientists during their daily and scientific routines on their missions within the extraterrestrial habitat and provide emotional support. During a week-long so-called analog mission with four scientists using a Wizard of Oz prototype, we derived design guidelines for such CUIs. Successively, based on the derived guidelines, we present the implementation and evaluation of two CUIs named CASSIOPEIA and PEGASUS.

\section{CCS CONCEPTS}

- Computer systems organization $\rightarrow$ Embedded systems; $R e$ dundancy; Robotics; • Networks $\rightarrow$ Network reliability.

\section{KEYWORDS}

Interactive Spaces, Conversational User Interfaces, Design Guidelines, Extraterrestrial habitats, Mars and Moon Mission, Human Space Exploration 
ACM Reference Format:

Ana Rita Gonçalves Freitas, Alexander Schülke, Simon Glaser, Pitt Michelmann, Thanh Nguyen Chi, Lisa Schröder, Zahra Fadavi, Gaurav Talekar, Jette Ternieten, Akash Trivedi, Jana Wahls, Warda Masood, Christiane Heinicke, and Johannes Schöning. 2021. Conversational User Interfaces to support Astronauts in Extraterrestrial Habitats. In 20th International Conference on Mobile and Ubiquitous Multimedia (MUM 2021), December 5-8, 2021, Leuven, Belgium. ACM, New York, NY, USA, 10 pages. https: //doi.org/10.1145/3490632.3490673

\section{INTRODUCTION \& MOTIVATION}

The monotonous living conditions in space were identified as one of the main causes of disturbances in the mental well-being of crew members [16]. Recently, researchers also found impairments on cognitive processes in participants who were immobilized and isolated [8]. As a result, the subsequent isolation and frustration, as well as the lack of a familiar environment and sensory input on spaceships or outposts, could endanger the mission's success and the crew's safety. In space missions that last a couple of months, those challenges were often addressed by communication with mission control and psychologists [47]. However, this is no longer possible on a 2-3 year-long mission to Mars due to message transmission times of at least 20 minutes in one way.

Interactive technologies are one critical success factor for such challenging missions. From Ubicomp technologies in mission control centers [24] to autonomous robots [2] many different technologies are used today to support astronauts and scientists. The crew module of the recently tested Dragon 2 is equipped with a variety of interactive touch screens for the astronauts to control the rocket. Nevertheless, not only the spacecraft are becoming interactive spaces and the habitats that the astronauts will inhabit on their increasingly long missions that last for multiple years. Interactive technologies that augment the astronauts in maintaining mental well-being and enriching their experiences are therefore as the basis for future space missions.

We believe that CUIs can play in important role in this space as communication is crucial for space missions [15]. NASA explored in 2005 a voice enabled procedure browser for the International Space Station (ISS) [44]. Today, astronauts at the ISS are being supported by CIMON, a CUI in space [5]. Its Russian counterpart, the humanoid robot Fedor ${ }^{1}$, was also designed to assist the astronauts on ISS. Such CUIs are supposed to assist astronauts on their challenging long-duration missions, in particular, to support them in research-related tasks during space flight or planetary exploration missions.

This paper presents the design, implementation, and evaluation of two CUIs for extraterrestrial habitats. Extraterrestrial habitats offer additional challenges for the design of interactive technology within them. The first habitat prototypes are very complex, and astronauts will require support from mission control to fulfill their tasks and duties. Nevertheless, direct communication will not (always) be possible (e.g., due to the moon's shadow or long distance to Mars). Therefore, we explore in this paper how CUIs can enrich the astronaut's experiences. To derive design guidelines for CUIs for extraterrestrial habitats, we performed a Wizard of $\mathrm{Oz}$

${ }^{1}$ short for Final Experimental Demonstration Object Research: https://tass.com/science/ 918017 study $n=4$ with a CUI within a prototype of an extraterrestrial habitat called MaMBA (Moon and Mars Base Analog, see figure 1) designed to serve humankind as an outpost on the Moon or Mars. The MaMBA [21] project aims to build a first functional extraterrestrial habitat prototype. Following the argument of [28] that such interfaces introduce a significant change in information access, we believe that it is essential to investigate how CUIs can complement interactive spaces in extreme conditions. Therefore, we explore which interfaces are suitable for extreme interactive spaces and illustrate this by developing two CUIs. With three explorative user studies, we found out that CUIs are heavily used in extraterrestrial habitats compared to other environments such as living rooms or office environments. Besides helping answering mission-specific requests, CUIs were also used for leisure and fun activities. From these conclusions, we derive four design guidelines for CUIs in space. Based on the guidelines, we report on the implementation and evaluation with a total of 22 users of two novel CUI concepts named CASSIOPEIA and PEGASUS (see figure 4). Both prototypes were evaluated within the habitat. For each CUI prototype for the evaluation, we focus on a particular aspect of the CUI. In the CASSIOPEIA study, we show that an extension of the CUI with a GUI helped the scientists to complete their tasks more efficiently. Even so, we measure no significant differences in terms of usability to a CUI only condition of CASSIOPEIA. In addition, the placement of such CUI within the habitat is important. With the PEGASUS study, we show that a CUI can ease the work tension and promote the well-being of the crew members by creating an emotional layer in conversation.

We contribute to the exploration of how research in $\mathrm{HCI}$ and the fast-developing field of space exploration can stimulate each other, as we envision a significant impact of $\mathrm{HCI}$ research for the success of future space missions (we refer to the crew module of Dragon 2 as an example). We are doing so by developing two novel CUI to assist the astronauts with their daily tasks and duties and enrich the UX in the space habitat. Following a user-centered approach with three user studies and the implementation of two novel CUI prototypes, we gained first knowledge of how to design CUIs for extraterrestrial space habitats. Examining our work presented in this paper with the lens of Oulasvirta et al. [40], we believe that our paper provides solutions to the conceptual and constructive problems when it comes to the development of CUI in extreme interactive spaces (e.g., an extraterrestrial habitat) and can inspire other researchers when developing CUIs for extreme environments. Oulasvirta et al. [40] describe scientific progress when developing novel technologies (including related fields) as improvements in the ability to solve significant problems related to human use of computing. Extending Laudan's philosophy of science [33], they defined HCI research as problem-solving, where the solution results in an improvement to problem-solving capacity. They describe that problems can be empirical, conceptual, or constructive, and the solution can be defined in terms of significance, effectiveness, efficiency, transfer, and confidence. We believe that novel technologies such as CUIs can play an essential role in mission success as they can provide emotional support or better assist the scientists in following their often very complex routines during the missions. On top of these contributions, we expect our research to apply outside this "lead user market" to novel technologies in terrestrial 
environments. Within MaMBA, we have the unique opportunity to research how human-centered methods perform in these extreme contexts.

The paper is structured as follows. First, after discussing related work, we describe a Wizard of Oz study and the derived guidelines. Based on that, we present the implementation of two novel CUI concepts named CASSIOPEIA and PEGASUS. This is followed by two evaluations of the systems, results, and discussion. Finally, the last section provides concluding remarks and an outlook on future work.

\section{RELATED WORK}

In this section we discuss the related work in the areas of mental health of astronauts and the use of CUIs in space A good overview on recent advances on research in CUI from various perspectives can be found in [13].

\subsection{Potential Measures to Support Astronaut Well-being during Long-term Missions}

Close cooperation between astronauts and flight surgeons, who are qualified to identify symptoms of possible behavioral problems, is essential in space missions [47] to maintain a good mental state for the astronauts [54]. Moreover, the certain possibility of a quick return to Earth at any time in near-Earth-missions, as well as the almost instantaneous communication between the astronauts and mission control are two additional important positive factors [47]. Together with professional psychological care, the astronauts of the ISS are often provided with packages from family and friends to be emotionally supported during space missions [47]. However, future space missions to Mars will require the astronauts to live in confined spaces for even longer periods of time (e.g. several years). In addition, communication with mission control will be limited due to time delays of up to around 20 minutes [47] one way. Thus the time between a message sent and the corresponding response is around 40 minutes. We expect such factors to decrease the social interaction between crew and mission control even more which acts as a negative factor on the mental health of the astronauts. Constant mental pressure, especially when the astronauts are confronted with failure, could endanger the missions and put their success at risk. First experiments were already conducted to study these effects in so-called analog missions to find out how to prevent human dysfunction during space missions. Research into technology that compensates for the lack of communication has already been conducted during those tests of human isolation in extra-terrestrial environments, which have all taken place on Earth so far [56]. Technologies have already proven themselves to be of great benefit in analog missions when used purposefully. The Mars 500 project simulated a 520 days lasting isolated Mars mission in a closed facility near Moscow [56]. In this mission, the six astronauts tested a technological system called EARTH, with which the astronauts could immerse themselves fully in several different relaxing digital environments like nature scenes, landscapes and parks. Another mission in an extreme environment where technology has proven to be beneficial is the HI-SEAS missions, which regularly take place in Hawaii. Six long-term missions have been conducted so far, although the last one had to be aborted after four

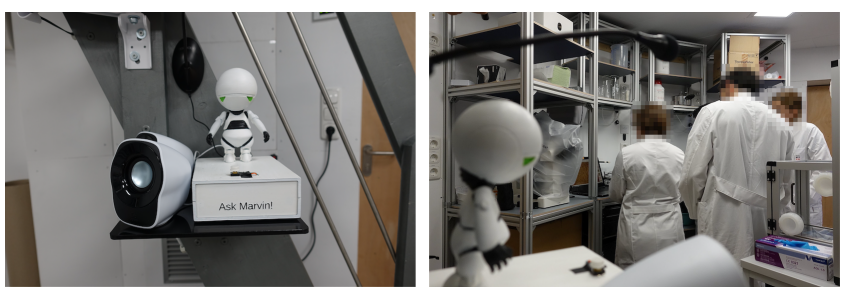

Figure 2: Wizard of $\mathrm{Oz}$ CUI, with which the four scientists in the extraterrestrial habitat prototype spent a week performing their experiments (left). Three of the fours scientists in the laboratory module of the habitat during the Wizard of Oz Study (right).

days because of an electric shock accident [22]. During one mission, scientists deployed ANSIBLE, a virtual world that allowed the participants and their families to interact with each other [57].

\subsection{Voice Assistants in Space}

CUIs such as Google Assistant, Amazon Alexa, and Siri are some of the most ubiquitous artificial intelligent systems that have been incorporated into our everyday lives. Despite having such a long development history [23, 29, 31], CUIs just recently became more and more ubiquitous [27] with the advent of devices like Google Home, Amazon Echo, and Apple HomePod in our living rooms. Even though guidelines for CUIs were developed decades ago [46],[26], the interaction with these devices is still complicated [28],[30]; therefore, general design guidelines for human-computer interaction (HCI) need to be improved [1],[19]. Recent research in the Ubicomp domain has been focusing on increasing learnability of CUIs [37], on designing CUIs to help people with overcoming problems caused by such interfaces when they arise [36], and on studying the differences between CUIs and classical graphical user interfaces (GUIs) to obtain the design patterns and optimise CUIs' effectiveness [52] or also on how to use CUI to assist in lab work [10]. Similarly, Murad et al. [35] discuss a first taxonomy of design guidelines for hands-free speech interfaces. While the studies on understanding the ways people interact with CUIs in everyday scenarios are very recent [29],[41], [30], [45], [49], [32], there is only a small amount of research on how CUIs can be used in other (more extreme) environments. As previously stated, a notable example of a conversational robot in space is CIMON, which was installed in the ISS for 14 months. CIMON had been provided with a clearly defined character and was configured with three different personality traits: serious, assisting and friendly, with the goal of being identified not only as an assistant [5].

\section{PRE-STUDY}

To identify the requirements of a CUI for an extraterrestrial habitat, a Wizard of $\mathrm{Oz}[17,38]$ experiment was conducted within one week in June 2019 inside the laboratory module of a MaMBA space habitat (see figure 2). The habitat is developed at the Center of Applied Space Technology and Microgravity (ZARM) in Bremen, Germany and comprises six connected but independent modules. In its final state, the habitat is meant to test crucial technologies such as life support, power systems, and remote communication [21]. A 
mock-up of the first module - the laboratory module - was used as the testbed for the study. The laboratory module is considered to be the center of the scientific habitat and is designed for simultaneous work of up to four scientists. Its floor layout is that of a circle with a diameter of $4.40 \mathrm{~m}$ which is divided into 18 segments. At the moment, the module is stand-alone but in its final design, it will feature three connections to the remaining modules of the habitat. There are three distinct working areas and one additional glove box (see figure 2). Typical lab work involves analyses of geological and biological samples and the preparation and conduction of experiments under conditions found in a lunar laboratory. The laboratory provides us with an ideal testbed as it allowed us to test CUIs with scientists in an extraterrestrial habitat prototype. During our Wizard of $\mathrm{Oz}$ study, a crew of four scientists performed biological, geological, and materials science experiments in the module over one week. The affordances of such CUIs in extraterrestrial habitats differ from the ones in a space station orbiting the Earth (e.g. ISS). The Wizard of Oz prototype of the CUI consisted of a Raspberry Pi running RaspbianOS with speakers and a microphone (see figure 2) placed in a box beside the main door, 1-3 meters away from the main working areas and a 3D printed figurine on top. The data from the microphone is transmitted to the wizard interface running on a laptop outside the habitat. The wizard can respond to the requests of the scientists by either using predefined templates offered in the interface or, in the case there is no template available, by inserting messages manually that are converted to speech through the SVOX Pico text-to-speech library.

\subsection{Participants}

Four scientists ( 2 females, 2 males, between 28 and 43 years old, 34 years old on average) were selected to prepare experiment protocols before the test run and to perform those experiments during the test run. All scientists are researchers with significant experience in laboratory work on space-related topics; three of them have a $\mathrm{PhD}$ in their respective field and the other one is an advanced $\mathrm{PhD}$ student.

\subsection{Procedure}

The test run was divided into nine sessions, each about typically $2.5 \mathrm{~h}$ long (with some variation: shortest was just slightly over an hour). All participants were briefed in the use of the habitat laboratory and safety protocols on the day before the first session. On the final day, after the last session, the scientists filled in questionnaires and were interviewed by one of the authors of this study. The scientists answered questions related to the usability and usage of the CUI as well as a larger catalog of mission specific questions. We logged all interactions (activations of the CUI as well as transcript the dialogues) with the Wizard of Oz prototype of the CUI and the sessions were recorded on video using two video cameras installed in the habitat. Using an iterative coding process, two independent coders transcribed and categorized all conversations into a schema described later in the results section. We defined an activation as a request initiated by either the scientists or the CUI, while a conversation is an activation which has at least one follow-up interaction. Unlike other commercial CUIs, our Wizard of Oz prototype could also initiate conversations; nevertheless, $50 \%$ of the activations were

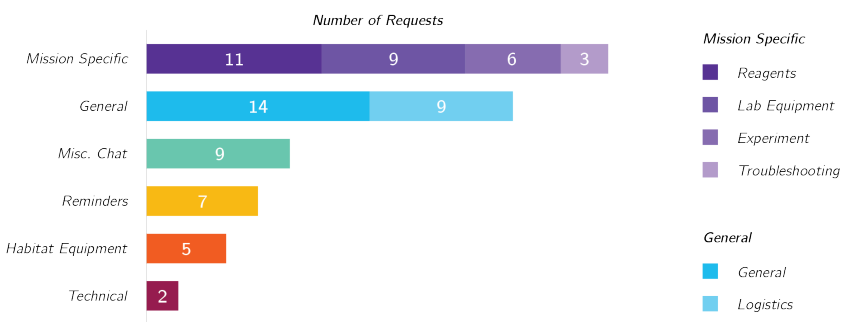

Figure 3: Categories of conversations with the Wizard of $\mathrm{Oz}$ prototype over the study period.

initiated by users, proving that the Wizard of $\mathrm{Oz}$ prototype was also proactively used by all the scientists.

\subsection{Results}

The data collected from the nine sessions stretches over roughly 20 hours. Within the nine sessions, we observed a total of 84 activations, out of which 75 were considered as conversations. The duration of the conversations largely varied. While the shortest conversation lasted just about 5 seconds (consisting of a simple command and execution), the longest one took 18 minutes (troubleshooting the VPN access). The median conversation duration was approximately 50 seconds.

(1) Mission-specific questions:

- Details about the experiment protocols (conversations related to the scientific experiments the scientists performed)

- Equipment utilized in each experiment (e.g. requesting the status or detailed information of a specific piece of equipment in the habitat)

- Chemical reagents required for each experiment (e.g. requesting information regarding their composition or other facts)

- Troubleshooting requests (e.g. rebooting a system or computer).

(2) General \& logistical questions:

- General (conversations that contain a question that the CUI could solve by inquiring the internet or its knowledge database)

- Logistical (logistical questions regarding the Wizard of $\mathrm{Oz}$ experiment)

(3) Miscellaneous chats (conversations with the CUI with no clear goal or task to complete, e.g. playing music)

(4) Reminders (conversations triggered by the CUI with alerts to the scientists)

(5) Configuration of settings of the extraterrestrial habitat (e.g. switching or dimming on the lights or controlling sensors in the habitat)

(6) Adjustment of settings of the CUI (e.g changing the volume of the speakers)

Figure 3 shows the distribution of the conversations for each category. While mission-specific questions cover a major part of the total requests (accounting for $52 \%$ of the total conversational 
duration or $34 \%$ of the total number of activations), non-missionrelated requests e.g. playing music or simply chatting with the CUI were also actively used. The troubleshooting conversations took the longest (one was the eighteen-minute conversation mentioned above), whereas reminders and configuration requests were rather short (around 10 to 15 seconds). The qualitative feedback from the interviews with all four scientists was that the CUI was fun to use and very helpful ("all my questions were answered"). The crew members also stressed that the CUI was very helpful when working with gloves. They unanimously agreed that CUIs would be useful in a "real" mission, as they could be operated hands-free while performing the experiments in the extraterrestrial habitat. The participants also proposed to have multiple different CUIs in the space habitat; e.g. a personal companion or sidekick and a general main intelligent CUI to support the whole crew and mission.

\section{DESIGN GUIDELINES FOR A CUI IN EXTRATERRESTRIAL HABITATS}

Based on the study and data presented in the previous section, we derived the following four main design guidelines for CUI in extraterrestrial habitats following the approach described in [42]:

\subsection{Mission-Specific \& Non-Specific Requests}

During the study, one of the very first activations by one of the scientists was a request to play some music for entertainment, while the scientists were setting up their experiments. Many other nonspecific requests were made by the astronauts during the study period. Thus, we believe, it is important to design not only for mission-specific requests but also for non-mission-critical tasks.

\subsection{Active Use}

The CUI was very actively used during our study by all four scientists. The study lasted solely a week, however, we did not witness any drop in usage over that period. Therefore, when designing CUI for extraterrestrial habitats, it should be noted that such interfaces are used more often compared to similar interfaces in other contexts. In addition, it is important to consider that the conversations also lasted longer compared to CUIs in other contexts [50]. While this could be an artifact of the study design, we believe that the next generations of CUI should enable more sophisticated conversations, not only in extraterrestrial habitats but also in all other contexts.

\subsection{Offering Multiple Modalities \& Two-Way Interaction}

It is useful to have means of interaction that allow continuing the conversation using other modalities than speech. For example, during the eigtheen-minute-long conversation, it would have been helpful to show some data on a screen attached to the CUI or send the scientist further information e.g. via mission control. Schaffer [48] also recently argued that this makes CUIs more robust. Moreover, compared to other usage contexts of CUIs, it also proved useful as a way for mission control to ask the astronauts to perform some tasks in the extraterrestrial habitat (e.g. restarting a computer that mission control had lost connection to, etc.).

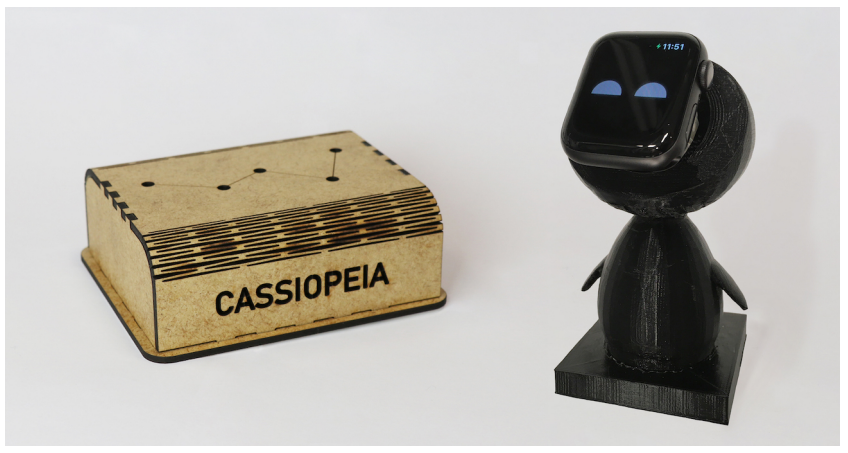

Figure 4: The CASSIOPEIA (left) and PEGASUS (right) prototypes.

\subsection{CUI As a Team Member}

Lastly, it is important to consider, that the scientists quickly adapted the CUI as a team member. The CUI can play a critical role in supporting astronauts on their often long and challenging missions [39]. It should also be mentioned that the scientists largely interacted with the CUI as they would with another person, most notably acknowledging receipt of a message by thanking him.

\section{CASSIOPEIA \& PEGASUS}

Based on the guidelines, we designed and implemented the two CUIs named CASSIOPEIA and PEGASUS for use in an extraterrestrial habitat as also proposed by the scientists from the Wizard of $\mathrm{Oz}$ study. Both were designed to cover all four design guidelines, but CASSIOPEIA was designed to support running experiments (focusing on the third guideline), PEGASUS was designed more as a personal companion that helped each astronaut throughout the day (focusing on the fourth guideline) as recommended in the study as well.

\subsection{CASSIOPEIA}

Unlike most current commercial systems that have been offering features that handle all of the non-critical mission requests, the proposed system - CASSIOPEIA - focuses on providing missionspecific assistance during executions of scientific experiments in an extraterrestrial habitat similar to [6]. Routinely throughout a mission, scientists are required to strictly follow predefined experiment protocols that typically include a short description of the experiment, a detailed list of the required reagents and equipment, and a step-by-step explanation of how to fulfill the experiment. These mandatory protocols may demand the registration of a report at the end of each scientific experiment by the scientist(s) involved, and optionally, the report can be sent to mission control or saved locally on the system once finalized. Besides acting as an experiment assistant, CASSIOPEIA also plays the role of a central system that stores all public information of the habitat, i.e. crew member list, experiment description, reagents, equipment, and instructions. This data is accessible and controllable by mission control, therefore, CASSIOPEIA also provides a communication channel between mission control and the habitat. The proposed CUI is able to handle all experiment-related requests coming in a variety of duration 


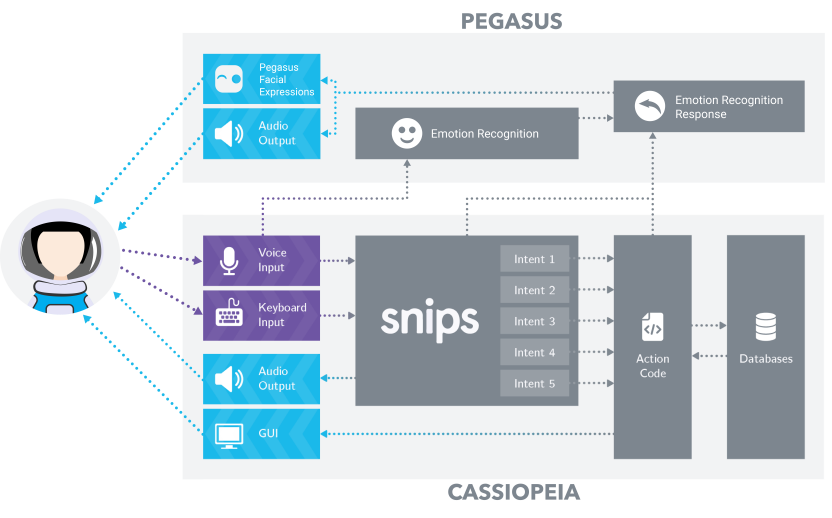

Figure 5: System architecture with I/O channels and core elements of CASSIOPEIA (bottom) and PEGASUS (top).

and complexities. In order to enable that, CASSIOPEIA utilizes a state machine to keep track of the ongoing process of selecting and following the step-by-step description of a procedure in order to ensure that the user stays on the correct path. Based on the current state, the system is able to predict and adapt its guidance to the crew members' intentions so as to minimize the crew members' confusion and frustrations when interpretation problems arise. As mentioned in the previously presented design guidelines, incorporating multiple modalities in CUIs can enhance the quality and experience of the human-computer interaction [53]. Therefore, CASSIOPEIA was developed to allow interaction with a GUI. The GUI provides clear suggestions of the available commands that are accessible in each stage of the interaction through subtle visual cues, which helps to guide the crew members through the process as well as to familiarize them with the eligible operation syntax and options. As suggested by Zamora [58], voice interaction shows most potential in cases where the user's hands are busy and therefore cannot interact with a GUI, which is the case for executing experiment-related activities. On the other hand, typing is considered to be more appropriate for tasks that require a higher level of detail, hence the system should offer the possibility to switch between voice-only and voice-and-visual modes to accommodate specific situations.

\subsection{PEGASUS}

In contrast to the central system CASSIOPEIA, PEGASUS (Personal Guiding Sidekick) is an interactive, helpful and emotional support companion. To a certain degree, it is an attentive system by remembering what it was told. Thus it is able to refer to the personal information and interests of the users to make the dialogue more personal. PEGASUS is embodied by an Apple Watch 5th edition and always displays one out of 16 different faces to support its responses and statements towards the user. The smartwatch is mounted onto a 3D figurine. The smartwatch shows different emotional facial expressions during conversations, such as happy, sad, laughing, surprising [43]. Physical and visual cues make the user more emotionally expressive and the figurine is perceived friendlier by the user and aides to benefit the crew member personality of the PEGASUS system [11],[14].

\subsection{Implementation}

An overview on the architecture of CASSIOPEIA and PEGASUS can be seen in figure 5. Both use the same underlying technical system and can share building blocks. For the experiments CASSIOPEIA and PEGASUS were designed to work independently from each other. CASSIOPEIA runs on a Raspberry Pi using one input channel (audio through the built-in microphones of the Raspberry $\mathrm{Pi}$ ) and two output channels (audio through the built-in speaker of the Raspberry Pi and visual through a monitor connected to the Raspberry Pi). An overview of the system's architecture can be seen in figure 5 and a photo of the CASSIOPEIA system can be seen in figure 4 . The audio output is created using an on-device textto-speech library called Hermes, while the visual output is offered simultaneously through the GUI displayed on a connected monitor. We strive for a seamless hybridization between the two output channels so as to minimize the latency between the progress indicator on the GUI and the narrative guidance. CASSIOPEIA processes audio inputs using the natural language processing engine SNIPS (https://snips.ai). The platform allowed us to create custom intents to train a speech model by submitting specific example sentences. SNIPS was discontinued on 31st January, 2020. However, due to the fact that all intents and speech model were created before that date, it is still possible to use CASSIOPEIA with any Raspberry Pi. Using the trained model, the system is able to detect intents from voice commands and once an intent is recognized, CASSIOPEIA runs a corresponding applet (designated as action code in figure 5). Each intent's action code will be executed to perform a certain task. The connection to the necessary services (e.g. databases, GUI synchronizer) will be initiated from the action code when required. Identical to CASSIOPEIA, PEGASUS makes use of the SNIPS framework to recognize natural language and user intents. Action codes associated with the different intents are executed and send text and face instructions to the Apple Watch via a web server. The user can interact with the CUI by saying the wake-word of the system, followed by a command. The audio is recorded via a microphone. The response is played by the smartwatch as soon as the user's intent is recognized and the face gets updated. As long as the dialogue expects another response from the user, the watch sends a signal after finishing its speech instructions in order to listen again.

\section{STUDY CASSIOPEIA}

The goal of the study was to investigate the effects of multi-modal interaction with CASSIOPEIA through an additional GUI. We investigated how it can help to guide scientists through experimental routines.

\subsection{Participants}

To examine the interaction between the user and CASSIOPEIA, we conducted a between-subject user study with 12 participants ( 2 female, 9 males, and 1 non-binary; between 21 and 32 years of age) inside the laboratory module of the habitat in February 2020. All of them were familiar with commercial voice assistants such as Google Assistant or Amazon Alexa, yet only approximately $25 \%$ of the participants shared that they had much experience with such technology. 


\subsection{Procedure}

The participants were randomly assigned into two groups to perform the same laboratory experiment procedures with either of the following setups: the participants used the CASSIOPEIA system with (CUI+GUI) or without (CUI-ONLY) an additional GUI, but the amount of information was consistent within both conditions. For both groups, the system was positioned in a central location within the laboratory module, that could easily be engaged with from any part of the module. The GUI was placed on a swivel mount next to it. Furthermore, the participants were not informed about the experiment procedure and the equipment and material in advance. They were instructed on which scientific experiment to select only before starting the interaction with our system. At this moment, they started the interaction by waking up the system with the command "Hello" which would give them a short introduction of what the system can do and some of the possible commands they can use to advance in the process. After completing the task, the participants were asked to fill out a questionnaire and take part in the follow-up interview.

To measure the usability of our assistant we used the System Usability Scale (SUS) [9] and the Post-Study System Usability Questionnaire (PSSUQ) [34]. In addition, the PSSUQ can be divided into sub-scales which evaluate system usefulness, information quality and interface quality. To further analyze the user expectations and behaviors we carried out one follow-up interview session after each experiment.

\subsection{Results}

As one participant was not able to complete the experiment we only report on the feedback of the 11 successful participants. The total time spent on each experiment differs significantly between CUIONLY and the CUI+GUI condition $(\mathrm{p}=0.028)$ using paired samples t-tests. The experiments were accomplished faster on the CUI+GUI $(618 \pm 92$ seconds) in comparison to the CUI-ONLY $(863 \pm 270 \mathrm{sec}-$ onds). The CUI-ONLY-Group showed a much higher divergence than the CUI+GUI-Group, which suggested that the influence of the CUI-ONLY on users' adaptability and performance was lower than that of the CUI+GUI. Users of the CUI-ONLY condition had to call the assistant significantly more often than those of the CUI+GUI condition $(p=0.02)$. Users of the CUI-ONLY-group asked the assistant to repeat 3.2 on average, while users of the CUI+GUI condition only asked to repeat one time.

The CUI-ONLY condition has a slightly higher SUS-Score $(M=74.6$, $\mathrm{SD}=18.7)$ than the CUI+GUI Condition $(\mathrm{M}=73.5, \mathrm{SD}=15.0)$. Both scores signify a good usability [3]. A paired samples t-test showed no significant difference between the SUS scores of the two conditions ( $\mathrm{p}=0.852$ ). The average PSSUQ score for the CUI-ONLY condition is $5.1(\mathrm{SD}=1.5)$ and for the CUI+GUI condition $5.4(\mathrm{SD}=1.2)$. Again, testing revealed no significant difference between the average PSSUQ scores in both conditions $(t(4)=-0.345, p=0.748)$. Both scores indicate that the system usefulness is slightly better than a neutral system. The evaluation of the sub-scales of the PSSUQ yielded no significant difference between the two conditions as well as no significant deviation from the calculated overall PSSUQ score.

During the experiment, most of the participants were able to adapt to the system and thus found it increasingly easy to interact with it over time. Overall, only 2 participants stated to be overwhelmed by the amount of information given at once and would have preferred smaller steps. In the interviews more than half of the participants of the CUI-ONLY stated that the available commands were intuitive and clear and 7 out of 11 participants found the system easy to use or becoming easier to use over time. 3 out of 6 participants that worked with the CUI+GUI prototype, remarked that the GUI should show images or figures to make certain steps of the experiment clearer or as a support in case a participant does not know a certain word. As described above we left the information in both conditions the same to ensure comparability. It is interesting to note that the placement of the CUI was of importance for the participants. 7 out of 11 participants would have preferred the system to be in front of them rather than next to them. As the lab space is very restrictive in the extraterrestrial habitat, participants had more freedom within the CUI-ONLY condition and in the CUI+GUI condition. It is also noteworthy that one participant with the lowest experience with voice assistants from the CUI-ONLY-group had the most trouble interacting with CASSIOPEIA. The participant with the lowest voice assistant experience from the CUI+GUI group, however, had a very good experience. This indicates that inexperienced users could profit from having a GUI combined with a voice interface.

\section{STUDY PEGASUS}

Within the second study, our goal was to investigate if a characterized PEGASUS prototype can help to establish an emotional connection between the CUI and the user. We performed a user study to explore the effect of a characterized PEGASUS version $(\mathrm{CH})$ compared with a non-characterized version of PEGASUS $(\mathrm{NCH})$ on the users mood and composure during an experiment. While the responses of the characterized CUI aim to simulate the behavior of a crew member, the non-characterized CUI only responds in an objective manner and provides information fast and efficiently. The experiment required each participant to actively inquire about the next steps from the CUI.

\subsection{Participants}

We conducted a between-subject user study with 10 participants ( 5 female, 5 males; between 23 and 30 years old) inside the laboratory module of the habitat in late February 2020. The group of participants was different from the previous two experiments, but more than half of the participants had previous experience using voice assistants.

\subsection{Procedure}

The participants were asked to build a small electromagnet within our space habitat guided by PEGASUS. The assembly involved many small and tedious steps so that the participants were forced to interact with PEGASUS. In contrast to the first experiment, the CUI was placed on the workbench of the participants next to the assembly area. In the $\mathrm{CH}$ condition the CUI always provided answers in respect to its character model and added additional comments to help the participants to carry out the experiment and loosen up their mood. Furthermore, the companion adapted its facial expression. We again used the Post-Study System Usability Questionnaire 
(PSSUQ) [34] as well as NASA-TLX [20] as quantitative measures as well as the short-form of the positive and negative affect schedule (PANAS)[55]. This was followed by semi-structured interviews after completion of the task.

The conversations between PEGASUS and the participants were transcribed to categorize different verbal cues, such as acknowledgments, initiating conversations, recalling conversations, expressing past content, excuse/apologize, joke, praise and small talk. Calculation of relative values, such as the percentage of requests in the form of questions or the count of "thank you" was done per total inquiries [18].

\subsection{Results}

The extent to which the participants felt excited was significantly different between the two conditions according to a WilcoxonMann-Whitney test $(W=1.5, p=0.022$ ) (see figure 6,right). While this also resulted in a higher task load while using the $\mathrm{CH}$ version $(42, S D=28.3)$ we could not show significant differences with a Wilcoxon-Mann-Whitney test $(p=0.2948)$ compared to using the $\mathrm{NCH}$ version $(29, S D=9.09)$. In the $\mathrm{CH}$ condition, PEGASUS caused wide spread task load results compared to the $\mathrm{NCH}$ version with a minimum value of 5.0 and a maximum value of 84.0. Participants with a high emotional connection to PEGASUS also rated the CUI more sympathetic. The experience with voice assistants, however, did not influence the sympathy towards the CUI. Unlike the condition, which did not influence the sympathy towards the CUI, the stress level of the participants shows a correlation with sympathy $(p=0.043)$. The emotional connection with the CUI shows such a correlation as well $(p=0.037)$. Figure 6 (left) visualizes these results and shows that low emotional connection and sympathy tend to result in higher stress levels during the experiment. Since the groups' sample sizes are small, the result should be treated carefully. Such a correlation cannot be observed by comparing the emotional connection with the NASA TLX workload. The interview sessions gave useful insights into how the participants felt during the whole process. There was a divided response among the participants. While some of them liked how the $\mathrm{CH}$ version was friendly towards them and asked personal information from the user to get to know them, some of them felt uncomfortable answering questions about themselves. One participant said, "I felt awkward telling my personal information to a digital entity". The majority of the participants regarded the physical body and the facial expressions as "appealing" and "attractive", which helped them to connect with the voice assistant better. Another participant said: "The figure was neat, I thought it's a penguin shape. I enjoyed how it looked with all the pleasant expressions and the tangible body." During the interview sessions it became clear that opinions on the characterized CUI can vary concerning each individual. However, the difference in participants' experiences with voice assistants did not show any significant influence.

\section{DISCUSSION}

We performed two user studies with very diverse CUIs within a laboratory module of an extraterrestrial space habitat. Most participants liked both CUI concepts and were able to complete the tasks given to them in both experiments. We focused to investigate effects
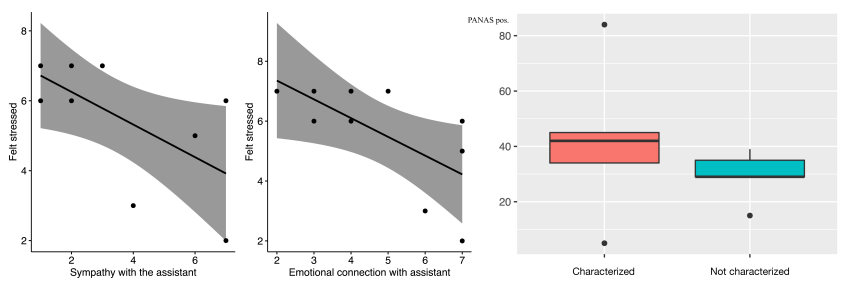

Figure 6: Correlations between questionnaire responses (left). PANAS excitement for the $\mathrm{CH}$ (red) and the NCH version of PEGASUS (green) (right).

of multimodality and emotional connection with CASSIOPEIA and PEGASUS rather on reducing crew time [59]. The number of test subjects is always somewhat smaller in experiments under "more extreme conditions." Therefore, the quantitative results are only valid to a limited extent. Nevertheless, we think that the iteration of the prototypes over a total of 3 studies provides insights into how CUIs can be designed for space habitats.

Even though the participants of the CASSIOPEIA CUI-ONLY condition took significantly longer to complete the experiment, we saw no difference regarding the SUS and PSSUQ. Participants of the CUI-ONLY condition also had to call the system significantly more often. This indicates that even though the perceived usability does not differ between the conditions, the CUI+GUI version is more efficient. The interview provided many useful insights into how to improve our voice assistant. On the one hand, information provided by the system should be presented concisely to prevent the user from being overwhelmed by it. On the other hand, the user needs the option to gain more detailed information if needed. Using a GUI in combination with a CUI seemed to be a more effective way to conduct scientific routines and the participants reinforced by their feedback our design guidelines from the Wizard of Oz study.

In the future, offering detailed information about the equipment and reagents in the laboratory and habitat could be helpful for such an assistant system. A future assistant should provide as much information as possible. However, it should be noted that all the additional information provided should be optional so that the user will not be overwhelmed by it. It is also important to note that in the habitat laboratory's small space, the placement of CASSIOPEIA was crucial for the participants. Therefore, we also recommend having one central system as CASSIOPEIA and complementing it with a more personal system such as PEGASUS.

Considering the excitement and the level of interest among the participants for PEGASUS, the deployment of such a CUI in space missions shows the potential to provide incentives for the astronaut and thus prevent boredom and monotony. However, it is necessary to consider the novelty effect when preparing for this kind of scenarios and further research has to be conducted to generate more knowledge regarding this matter. CUIs with embodiment and facial expressions are still rare since the commercial market provides mostly assistants without visual clues that could provide an emotional layer in their responses. It turned out that all participants rated both versions of the system as very interesting. When evaluating the study, it is noticeable that there seems to be a connection between how stressed users were and how sympathetic the user 
rated the system. The statement is being supported by the fact that the emotional connection shows a significant correlation as well. This aspect requires further research with larger test groups to assure its validity. However, a positive effect on astronauts' stress level through technology would represent a convenient method to contribute to the astronaut's mental well-being.

One crucial question that remains unanswered in this context is how or when the emotional connection with the CUI gets created. Our data suggest that sympathy and an emotional connection do not arise from the mere characterization of the CUI. Other studies in this area suggest personality tests that are carried out in advance with each test person [7]. To achieve an emotional connection with the CUI, the system's character profile could then be adjusted to a personality matching the participants' personality.

\section{CONCLUSION \& FUTURE WORK}

In this paper, we described the user-centered design process of CUIs for extraterrestrial space habitats. Within three studies, we derived guidelines for such systems and implemented and evaluated two prototypes. With the first week-long Wizard of Oz study on the use of a CUI in a lunar habitat and by the qualitative feedback of the scientists, we derived four guidelines for the development of CUIs for extraterrestrial habitats. Based on that, we presented the implementation of such CUIs named CASSIOPEIA and PEGASUS by taking the derived guidelines as a basis. We performed two additional studies to investigate the effects of multimodality and emotional connection with these systems with both prototypes. Our results can inspire other researchers working on CUIs in other domains, especially in more extreme environments. Well-designed CUIs can help to increase mission success and enrich the experiences of the astronauts on those missions.

In the future, we will perform an additional study comparing CASSIOPEIA against the Wizard of Oz prototype to evaluate the proposed guidelines during a more extended mission (e.g., similar to the Wizard of $\mathrm{Oz}$ study period). We will also focus on the impacts of a CUI as a team member, as the lunar habitat with its small spatial footprint is challenging for teamwork. It could also be interesting to implement a personal CUI to serve astronauts' psychological and emotional needs better. We believe, as Bentley et al. [4] also noted recently, that it is necessary to carry out more long-term studies to test CUIs in very different environments [12] in order to adapt them to the needs of the users. We also believe that those systems are suited to support Parastronaut as well [51]. Surviving in an extraterrestrial environment with limitations in real-time communication and personal isolation can be a severe challenge. We believe that turning those environments into interactive spaces can help to protect the astronaut's well-being. PEGASUS, a friendly CUI companion to ease the work tension and to promote the wellbeing of the crew members, can help by creating an emotional layer in conversation. Our studies suggest that the deployment of a characterized CUI might require a prior matching process between the test subject's character and the provided character simulation to benefit from it. According to our study, those benefits can range from excitement and interest to significant drops in stress levels. For astronauts, these might be important factors to support their psychological well-being during their missions. Today, the euphoria for space exploration has awakened again, with plans for colonizing the Moon and Mars [25]. However, a human-crewed mission to Mars would require about 2.5 years or more. Consequently, it is even more important to actively support and augment the mental wellbeing and experiences of the astronauts as such missions can be extremely monotonous and cause cognitive decline, social isolation, and frustration. Interactive technologies such as CUIs can be a part of the solution to these problems.

The project is funded by the Klaus Tschira Stiftung gGmbH. The authors would like to thank M. Arnhof, L. Orzechowski, M. Stadtlander, R. Mairose, P. Prengel from ZARM, and the student team of the HCI lab at the University of Bremen funded by the Volkswagen foundation through a Lichtenberg professorship that helped set up the habitat laboratory for their contributions to the project. In addition, the authors would like to thank Y. Nahas for his contribution to his particular study.We would especially like to thank all the students and co-authors from the master project "Living on Mars" for carrying out the development of the CUIs and the studies. This work is open access thanks to the University of St. Gallen, Switzerland.

\section{REFERENCES}

[1] Saleema Amershi, Dan Weld, Mihaela Vorvoreanu, Adam Fourney, Besmira Nushi, Penny Collisson, Jina Suh, Shamsi Iqbal, Paul N Bennett, Kori Inkpen, et al. 2019. Guidelines for human-AI interaction. In Proceedings of the 2019 CHI Conference on Human Factors in Computing Systems. ACM, 3.

[2] Max Bajracharya, Mark W Maimone, and Daniel Helmick. 2008. Autonomy for mars rovers: Past, present, and future. Computer 41, 12 (2008), 44-50.

[3] Aaron Bangor. 2009. Determining What Individual SUS Scores Mean: Adding an Adjective Rating Scale. 4, 3 (2009), 10.

[4] Frank Bentley, Chris Luvogt, Max Silverman, Rushani Wirasinghe, Brooke White, and Danielle Lottrjdge. 2018. Understanding the long-term use of smart speaker assistants. Proceedings of the ACM on Interactive, Mobile, Wearable and Ubiquitous Technologies 2, 3 (2018), 91.

[5] Matthias Biniok. 2019. Project CIMON fournal, Stories about DLR's technology experiment CIMON. https://www.ibm.com/thought-leadership/smart/de-de/aiin-space/journal/index.html

[6] Dan Bohus and Alexander I Rudnicky. 2005. LARRI: A language-based maintenance and repair assistant. In Spoken multimodal human-computer dialogue in mobile environments. Springer, 203-218.

[7] Michael Braun, Anja Mainz, Ronee Chadowitz, Bastian Pfleging, and Florian Alt. 2019. At Your Service: Designing Voice Assistant Personalities to Improve Automotive User Interfaces. In Proceedings of the 2019 CHI Conference on Human Factors in Computing Systems (Glasgow, Scotland Uk) (CHI '19). Association for Computing Machinery, New York, NY, USA, Article 40, 11 pages. https: //doi.org/10.1145/3290605.3300270

[8] Katharina Brauns, Anika Werner, Hanns-Christian Gunga, Martina A Maggioni, David F Dinges, and Alexander Stahn. 2019. electrocortical evidence for Impaired Affective Picture processing after Long-term immobilization. Scientific reports 9 , 1 (2019), 1-9.

[9] John Brooke. 1996. SUS - A quick and dirty usability scale. (June 1996), 7.

[10] Julia Cambre, Ying Liu, Rebecca E Taylor, and Chinmay Kulkarni. 2019. Vitro: Designing a Voice Assistant for the Scientific Lab Workplace. In Proceedings of the 2019 on Designing Interactive Systems Conference. 1531-1542.

[11] Heloisa Candello, Claudio Pinhanez, and Flavio Figueiredo. 2017. Typefaces and the perception of humanness in natural language chatbots. In Proceedings of the 2017 CHI Conference on Human Factors in Computing Systems. 3476-3487.

[12] Fabio Catania. 2019. Conversational Technology and Affective Computing for Cognitive Disability. In Proceedings of the 24th International Conference on Intelligent User Interfaces: Companion (Marina del Ray, California) (IUI '19). ACM, New York, NY, USA, 153-154. https://doi.org/10.1145/3308557.3308723

[13] Hongshen Chen, Xiaorui Liu, Dawei Yin, and Jiliang Tang. 2017. A survey on dialogue systems: Recent advances and new frontiers. Acm Sigkdd Explorations Newsletter 19, 2 (2017), 25-35.

[14] Saemi Choi and Kiyoharu Aizawa. 2019. Emotype: Expressing emotions by changing typeface in mobile messenger texting. Multimedia Tools and Applications 78, 11 (2019), 14155-14172.

[15] Leigh Clark, Nadia Pantidi, Orla Cooney, Philip Doyle, Diego Garaialde, Justin Edwards, Brendan Spillane, Emer Gilmartin, Christine Murad, Cosmin Munteanu, 
et al. 2019. What makes a good conversation? challenges in designing truly conversational agents. In Proceedings of the 2019 CHI Conference on Human Factors in Computing Systems. 1-12.

[16] Yvonne A Clearwater and Richard G Coss. 1991. Functional Esthetics to Enhance Weil-Being in Isolated and Confined Settings. In From Antarctica to outer space. Springer, 331-348

[17] Nils Dahlbäck, Arne Jönsson, and Lars Ahrenberg. 1993. Wizard of Oz studies-why and how. Knowledge-based systems 6, 4 (1993), 258-266.

[18] Jasper Feine, Ulrich Genewuch, Stefan Morana, and Alexander Maedche. 2019 A Taxonomy of Social Cues for Conversational Agents. In International fournal of Human-Computer Studies (International fournal of Human-Computer Studies Volume 132). 138-161.

[19] Jonathan Grudin and Richard Jacques. 2019. Chatbots, humbots, and the quest for artificial general intelligence. In Proceedings of the 2019 CHI Conference on Human Factors in Computing Systems. 1-11.

[20] Sandra G Hart and Lowell E Staveland. 1988. Development of NASA-TLX (Task Load Index): Results of empirical and theoretical research. In Advances in psychology. Vol. 52. Elsevier, 139-183.

[21] Christiane Heinicke, Leszek Orzechowski, Rawel Abdullah, Maria von Einem, and Marlies Arnhof. 2018. Updated Design Concepts of the Moon and Mars Base Analog (MaMBA). In European Planetary Science Congress, Vol. 12.

[22] HI-SEAS. 2020. Hawai'i Space Exploration Analog and Simulation. http://hiseas.org/

[23] Eric Horvitz. 1999. Principles of mixed-initiative user interfaces. In Proceedings of the SIGCHI conference on Human Factors in Computing Systems. ACM, 159-166.

[24] Elaine M Huang, Elizabeth D Mynatt, and Jay P Trimble. 2006. Displays in the wild: understanding the dynamics and evolution of a display ecology. In International Conference on Pervasive Computing. Springer, 321-336.

[25] Benjamin Kading and Jeremy Straub. 2015. Utilizing in-situ resources and 3D printing structures for a manned Mars mission. Acta Astronautica 107 (2015), 317-326.

[26] Candace Kamm. 1995. User interfaces for voice applications. Proceedings of the National Academy of Sciences 92, 22 (1995), 10031-10037.

[27] Joseph'Jofish' Kaye, Joel Fischer, Jason Hong, Frank R Bentley, Cosmin Munteanu, Alexis Hiniker, Janice Y Tsai, and Tawfiq Ammari. 2018. Panel: voice assistants, UX design and research. In Extended Abstracts of the 2018 CHI Conference on Human Factors in Computing Systems. ACM, panel01.

[28] Julia Kiseleva, Kyle Williams, Jiepu Jiang, Ahmed Hassan Awadallah, Aidan C Crook, Imed Zitouni, and Tasos Anastasakos. 2016. Understanding user satisfac tion with intelligent assistants. In Proceedings of the 2016 ACM on Conference on Human Information Interaction and Retrieval. 121-130.

[29] Lorenz Cuno Klopfenstein, Saverio Delpriori, Silvia Malatini, and Alessandro Bogliolo. 2017. The rise of bots: A survey of conversational interfaces, patterns, and paradigms. In Proceedings of the 2017 Conference on Designing Interactive Systems. ACM, 555-565.

[30] Dounia Lahoual and Myriam Frejus. 2019. When Users Assist the Voice Assistants: From Supervision to Failure Resolution. In Extended Abstracts of the $2019 \mathrm{CHI}$ Conference on Human Factors in Computing Systems. ACM, CS08.

[31] James A Landay, Nuria Oliver, and Junehwa Song. 2019. Conversational User Interfaces and Interactions. IEEE Computer Architecture Letters 18, 02 (2019), 8-9.

[32] Walter S. Lasecki, Rachel Wesley, Jeffrey Nichols, Anand Kulkarni, James F. Allen, and Jeffrey P. Bigham. 2013. Chorus: A Crowd-powered Conversational Assistant In Proceedings of the 26th Annual ACM Symposium on User Interface Software and Technology (St. Andrews, Scotland, United Kingdom) (UIST '13). ACM, New York, NY, USA, 151-162. https://doi.org/10.1145/2501988.2502057

[33] Larry Laudan. 1978. Progress and its problems: Towards a theory of scientific growth. Vol. 282. Univ of California Press.

[34] James R. Lewis. 1995. IBM computer usability satisfaction questionnaires: Psychometric evaluation and instructions for use. International fournal of Human-Computer Interaction 7, 1 (Jan. 1995), 57-78. https://doi.org/10.1080/ 10447319509526110

[35] Christine Murad, Cosmin Munteanu, Leigh Clark, and Benjamin R Cowan. 2018 Design guidelines for hands-free speech interaction. In Proceedings of the 20th International Conference on Human-Computer Interaction with Mobile Devices and Services Adjunct. 269-276.

[36] Chelsea Myers, Anushay Furqan, Jessica Nebolsky, Karina Caro, and Jichen Zhu. 2018. Patterns for How Users Overcome Obstacles in Voice User Interfaces. In Proceedings of the 2018 CHI Conference on Human Factors in Computing Systems (Montreal QC, Canada) (CHI '18). ACM, New York, NY, USA, Article 6, 7 pages. https://doi.org/10.1145/3173574.3173580

[37] Chelsea M. Myers. 2019. Adaptive Suggestions to Increase Learnability for Voice User Interfaces. In Proceedings of the 24th International Conference on Intelligen User Interfaces: Companion (Marina del Ray, California) (IUI '19). ACM, New York, NY, USA, 159-160. https://doi.org/10.1145/3308557.3308727

[38] Youssef Nahas, Christiane Heinicke, and Johannes Schöning. 2019. MARVIN Identifying Design Requirements for an AI powered Conversational User Interface for Extraterrestrial Space Habitats.
[39] Clifford Ivar Nass and Scott Brave. 2005. Wired for speech: How voice activates and advances the human-computer relationship. MIT press Cambridge, MA.

[40] Antti Oulasvirta and Kasper Hornbæk. 2016. Hci research as problem-solving. In Proceedings of the 2016 CHI Conference on Human Factors in Computing Systems. 4956-4967.

[41] Martin Porcheron, Joel E Fischer, Stuart Reeves, and Sarah Sharples. 2018. Voice interfaces in everyday life. In proceedings of the 2018 CHI conference on human factors in computing systems. ACM, 640.

[42] Daniela Quiñones and Cristian Rusu. 2017. How to develop usability heuristics: A systematic literature review. Computer Standards \& Interfaces 53 (2017), 89-122.

[43] Boele de Ed Raad and Marco Ed Perugini. 2002. Big five factor assessment: Introduction. Hogrefe \& Huber Publishers.

[44] Manny Rayner, Beth Ann Hockey, Nikos Chatzichrisafis, Kim Farrell, and JeanMichel Renders. 2005. A voice enabled procedure browser for the International Space Station. In Proceedings of the ACL Interactive Poster and Demonstration Sessions. 29-32.

[45] Stuart Reeves, Martin Porcheron, Joel E. Fischer, Heloisa Candello, Donald McMillan, Moira McGregor, Robert J. Moore, Rein Sikveland, Alex S. Taylor, Julia Velkovska, and Moustafa Zouinar. 2018. Voice-based Conversational UX Studies and Design. In Extended Abstracts of the 2018 CHI Conference on Human Factors in Computing Systems (Montreal QC, Canada) (CHI EA '18). ACM, New York, NY, USA, Article W38, 8 pages. https://doi.org/10.1145/3170427.3170619

[46] Steven Ross, Elizabeth Brownholtz, and Robert Armes. 2004. Voice user interface principles for a conversational agent. In Proceedings of the 9th international conference on Intelligent user interfaces. Citeseer, 364-365.

[47] Nick Salamon, Jonathan M. Grimm, John M. Horack, and Elizabeth K. Newton. 2018. Application of Virtual Reality for Crew Mental Health in Extended-Duration Space Missions. In 68th International Astronautical Congress (Ohio, USA) (IAC). IAF, 25-29.

[48] Stefan Schaffer and Norbert Reithinger. 2019. Conversation is Multimodal: Thus Conversational User Interfaces Should Be As Well. In Proceedings of the 1st International Conference on Conversational User Interfaces (Dublin, Ireland) (CUI '19). ACM, New York, NY, USA, Article 12, 3 pages. https://doi.org/10.1145/ 3342775.3342801

[49] Alex Sciuto, Arnita Saini, Jodi Forlizzi, and Jason I Hong. 2018. Hey Alexa, What's Up?: A mixed-methods studies of in-home conversational agent usage. In Proceedings of the 2018 Designing Interactive Systems Conference. ACM, 857-868.

[50] Ingo Siegert, Julia Krüger, Olga Egorow, Jannik Nietzold, Ralph Heinemann, and Alicia Lotz. 2018. Voice assistant conversation corpus (VACC): A multi-scenario dataset for addressee detection in human-computer-interaction using amazon's ALEXA. In Workshop on Language and Body in Real Life \& Multimodal Corpora. Miyazaki, Japan.

[51] Jennifer Sills, Christiane Heinicke, Marcin Kaczmarzyk, Benjamin Tannert, Aleksander Wasniowski, Malgorzata Perycz, and Johannes Schöning. 2021. Disability in space: Aim high. Science 372, 6548 (2021), 1271-1272.

[52] James Simpson. 2019. How is Siri Different Than GUIs?. In Proceedings of the 24th International Conference on Intelligent User Interfaces: Companion (Marina del Ray, California) (IUI '19). ACM, New York, NY, USA, 145-146. https://doi. org $/ 10.1145 / 3308557.3308728$

[53] Bernhard Suhm, Brad Myers, and Alex Waibel. 2001. Multimodal error correction for speech user interfaces. ACM transactions on computer-human interaction (TOCHI) 8, 1 (2001), 60-98.

[54] Chiew Seng Sean Tan, Johannes Schöning, Kris Luyten, and Karin Coninx. 2014. Investigating the effects of using biofeedback as visual stress indicator during video-mediated collaboration. In Proceedings of the SIGCHI Conference on Human Factors in Computing Systems. 71-80.

[55] Edmund R Thompson. 2007. Development and validation of an internationally reliable short-form of the positive and negative affect schedule (PANAS). Fournal of cross-cultural psychology 38, 2 (2007), 227-242.

[56] Igor' Borisovich Ushakov, Member Boris Vladimirovich, Yuri Arkad'evich Bubeev, Vadim Igorevich Gushin, Galina Yur'evna Vasil'eva, Alla Gennad'evna Vinokhodova, and Dmitrii Mikhailovich Shved. 2014. Main findings of psychophysiological studies in the Mars 500 experiment. Herald of the Russian Academy of Sciences 84, 2 (01 Mar 2014), 106-114.

[57] Peggy Wu, Tammy Ott, and Jacki Morie. 2016. ANSIBLE: Social Connectedness through a Virtual World in an Isolated Mars Simulation Mission. In Proceedings of the 2016 Virtual Reality International Conference (Laval, France) (VRIC '16). Association for Computing Machinery, New York, NY, USA, Article 28, 4 pages. https://doi.org/10.1145/2927929.2927933

[58] Jennifer Zamora. 2017. I'm Sorry, Dave, I'm Afraid I Can't Do That: Chatbot Perception and Expectations. In Proceedings of the 5th International Conference on Human Agent Interaction. ACM, 253-260.

[59] Conrad Zeidler, Gerrit Woeckner, Johannes Schöning, Vincent Vrakking, Paul Zabel, Markus Dorn, Daniel Schubert, Birgit Steckelberg, and Josefine Stakemann. 2021. Crew time and workload in the EDEN ISS greenhouse in Antarctica. Life Sciences in Space Research (2021). https://doi.org/10.1016/j.lssr.2021.06.003 